\title{
Conditions-cadres générales et explications
}

\section{Conditions-cadres des négociations salariales}

Comme les sociétés cantonales de médecine élaborent depuis 1991 leurs propres recommandations de salaires pour les assistantes médicales, nous renonçons désormais à en publier pour l'ensemble de la Suisse. Les conditions-cadres ci-après conservent toutefois leur valeur pour fixer individuellement le salaire d'une assistante médicale.

\section{Formation}

Certificat fédéral de capacité d'assistante médicale ou diplôme de la Fédération des médecins suisses (estampille) avec autorisation de radiographies.

\section{Conditions de travail: bases de calcul}

- Semaine de 42 heures en moyenne annuelle

- 4 semaines de vacances (assistantes médicales de moins de 20 ans et de plus de 50 ans: 5 semaines).

Toute dérogation importante à ces conditions sera répercutée sur le salaire.

\section{Normes régionales}

Les salaires doivent être adaptés aux conditions régionales.

\section{4. $13^{\mathrm{e}}$ salaire}

Un $13^{\mathrm{e}}$ salaire doit être versé à l'assistante médicale à la fin de l'année. Si les rapports de service n'ont pas duré pendant toute l'année civile, le $13^{\mathrm{e}}$ salaire sera versé au prorata des mois d'engagement.

\section{Primes d'ancienneté et augmentations du salaire réel}

Le montant d'une prime d'ancienneté devrait figurer, également en 2018, parmi les points discutés au cours de l'entretien annuel sur les qualifications de l'employée. Au moment de définir le salaire, toute responsabilité supplémentaire (p. ex. formation des personnes en apprentissage) ou formation complémentaire (p. ex. en radiologie à fortes doses, etc.) de l'employée devrait être valorisée et le salaire adapté en conséquence.
Un modèle pour conduire un entretien de collaborateur est à votre disposition sur le site de la FMH, à la rubrique Assistantes médicales: http://www.fmh.ch/ $\mathrm{fr} /$ services/assistantes_medicales.html

\section{Compensation du renchérissement}

Le renchérissement devrait être compensé. Lorsque la compensation du renchérissement est convenue par contrat, elle doit être accordée en tous les cas. Si le renchérissement est négatif, ce dernier ne doit pas être compensé (ISPC septembre 2017: 0,9\%; octobre 2017: 0,9\%, indice de base: décembre 2015).

\section{Travail à temps partiel rétribué au mois}

Pour le travail à temps partiel, le salaire brut recommandé s'élève au $1 / 42$ du salaire brut entier multiplié par le nombre des heures de travail hebdomadaire convenu (base de calcul: 42 heures hebdomadaires).

\section{Salaire horaire}

Un salaire horaire est recommandé en cas d'horaire de travail très réduit et en même temps irrégulier. Le taux horaire conseillé est de 6\%o du salaire mensuel pour une activité à temps complet (le $13^{\mathrm{e}}$ salaire y étant inclus au prorata). Il sera complété par une contribution aux vacances de $8,33 \%$ correspondant aux quatre semaines de vacances habituelles (pour 5 semaines le taux sera de 10,64\% et pour 6 semaines de 13,04\% etc.), qui devra figurer séparément sur chaque bulletin de salaire. Ce mode de calcul est également valable pour le paiement des heures supplémentaires.

Pour éviter les désavantages par rapport à un engagement avec un salaire mensuel, nous recommandons de convenir d'une indemnisation des jours fériés. En vertu de la loi sur le travail, les cantons peuvent disposer de 8 jours fériés équivalents à des dimanches, auxquels s'ajoute encore le 1er $^{\text {er }}$ aout. Il s'agit donc au maximum de 9 jours fériés par an. Il faut cependant tenir compte du fait que certains jours fériés sont toujours à la même date (p. ex. le $1^{\text {er }}$ mai) et peuvent donc tomber un samedi ou un dimanche. De plus, les jours fériés diffèrent d'un canton à l'autre. De ce fait, pour éviter de refaire le calcul tous les ans pour chaque canton, on 
part d'une moyenne de 7,5 jours fériés annuels qui tombent sur un jour ouvrable.

L'indemnisation des jours fériés se calcule comme les indemnités de vacances sur la base de 260 jours de travail par an:

$7,5$ jours fériés $/$ ( 260 jours de travail $-7,5$ jours fériés $)=$ $2,97 \%$ du salaire horaire brut.

\section{Heures supplémentaires (chiffre 2 du contrat type)}

Dans la mesure du possible, les heures supplémentaires doivent être compensées par du temps libre ou d'éventuelles vacances supplémentaires (= vacances au-delà du minimum légal de 4 semaines) d'une même durée. Dans des cas exceptionnels, il est possible de choisir parmi les variantes suivantes:

- L'indemnisation des heures supplémentaires par une majoration de salaire de $25 \%$ pour une activité à temps complet ou à temps partiel (préconisée par la FMH / le SVA uniquement pour les employés à temps complet).

- Dans le cas d'une activité à temps partiel, les heures supplémentaires sont indemnisées selon le taux horaire normal dans la mesure où elles ne dépassent pas la durée du temps de travail réglementaire d'une assistante à temps plein au cabinet médical (préconisée par le SVA). ${ }^{1}$

- D'un commun accord écrit, l'employeur et l'assistante médicale peuvent choisir une autre réglementation.

Le taux horaire est de $6 \%$ o d'un plein salaire mensuel pour une activité à temps complet, auquel s'ajoute la contribution aux vacances (cf. chiffre 8 , "Salaire horaire»).

\section{Les dispositions et recommandations suivantes s'appliquent à tous les salaires:}

\section{Déductions sur le salaire brut}

- AVS, AI, APG, AC: 6,225\% (AVS, AI, APG = 5,125\%, AC = $1,1 \%)$ injustifié accordé aux employées à temps partiel. Lorsqu'une assistante médicale travaille au-del de son temps partie convenu (et jusqu'à hauteur du temps de travail réglementaire de ses collègues engagées à plein temps), elle a éventuellement droit à un supplément de salaire alors que ses collègues touchent un salaire normal.

\section{Explications concernant le contrat type}

\section{Durée du travail et saisie du temps de travail} (ch. 2 du contrat type)

Conformément à l'art. 46 LTr et à l'art. 73 OLT 1, l'employeur est tenu d'enregistrer le temps de travail de ses employées. Pour chacune d'elles, il doit indiquer de manière claire quand elle a travaillé, pris ses pauses et terminé son travail. Ces indications permettent de vérifier si les dispositions de la loi sur le travail concernant la durée du travail et le repos sont respectées. L'employeur est tenu de conserver l'enregistrement du temps de travail pendant 5 ans (art. 73 al. 2 OLT 1).

Un système électronique de timbrage ne constitue pas le seul moyen de saisir le temps de travail, d'autres possibilités ont également fait leurs preuves (p. ex. tableau Excel). Nous recommandons toutefois aux employeurs de viser les fichiers Excel lorsque l'assistante médicale saisit elle-même son temps de travail.

Un fichier Excel pour la saisie du temps de travail est à votre disposition sur le site de l'ARAM (Association romande des assistantes médicales) et du SVA (Schweizerischer Verband Medizinischer Praxis-Fachpersonen).

Nous recommandons de commencer à compter le temps de travail au moment de l'arrivée au cabinet avec les travaux de mise en place (p. ex. démarrage de l'ordinateur, aération de la salle d'attente ou rangement des journaux, levée du courrier, etc.), et non au moment de l'arrivée du premier patient. De la même manière, les travaux du soir comme le rangement, le nettoyage des instruments de travail, font partie du temps de travail, à l'inverse du trajet qui ne compte pas comme temps de travail (à l'exception du service de piquet, ch. $6.6 \mathrm{du}$ contrat type). L’employeur peut élaborer des directives pour définir un début de travail efficace.

Différents modèles de travail existent. Hormis le temps de travail hebdomadaire recommandé dans le contrattype, il est également possible d'annualiser le temps de travail. Cette méthode offre une plus grande marge de manœuvre dans l'organisation mais il faut cependant tenir compte de la limite des 50 heures hebdomadaires, et veiller à ce que la semaine de 50 heures ne devienne pas la règle. De plus, les changements importants par rapport à l'emploi du temps hebdomadaire habituel doivent être pris d'un commun accord entre les deux parties. Dans l'idéal, le compte temps des employées devrait être équilibré en fin d’année. 
En cas d'avoirs en heures supplémentaires, ceux-ci devraient être compensés par du temps libre au plus tard d'ici le milieu de l'année suivante. Il en va de même pour les jours de vacances.

\section{Demi-journée de congé (ch. 6.2 du contrat- type)}

Conformément à l'art. 21 LTr, l'employeur est tenu de donner au moins une demi-journée de congé par semaine à son employée lorsque le travail hebdomadaire est réparti sur plus de cinq jours. C'est une exigence minimale.

\section{Mères qui allaitent (ch. 6.7 du contrat type)}

Le temps nécessaire à l'allaitement doit être accordé aux mères qui allaitent et l'employeur doit mettre à disposition un lieu approprié pour l'allaitement au cabinet (art. 34 OLT 3). Pour un travail quotidien de 4 heures au plus, les mères qui allaitent ont droit à 30 minutes d'allaitement dans le cadre de leur temps de travail rémunéré, pour un temps de travail quotidien de 4 heures et plus, ce droit est de 60 minutes, et pour un travail quotidien de plus de 7 heures, de 90 minutes. Il s'agit à chaque fois de temps minimum. Indépendamment du fait que l'allaitement ait lieu sur place au cabinet ou en dehors du lieu de travail, l'employée dispose du même temps rémunéré pour l'allaitement. Si elle quitte le lieu de travail, aucune prolongation du temps rémunéré n'est prévue pour l'allaitement. Il est cependant possible de trouver un autre accord entre l'employeur et l'employée concernée.

\section{Protection de la santé pendant la grossesse (ch. 6.9 du contrat type)}

Par travaux dangereux o pénibles, on entend le déplacement manuel de charges lourdes; les tâches imposant des mouvements ou des postures engendrant une fatigue précoce; les travaux impliquant l'impact de chocs, de secousses ou de vibrations; les travaux exposant à la chaleur $\left(\max .28^{\circ} \mathrm{C}\right)$, au froid $(\mathrm{min}$ $-5^{\circ} \mathrm{C}$ ) et à l'humidité; les activités soumises aux effets de radiations nocives ou au bruit $\geq 85 \mathrm{~dB}$, les activités soumises aux effets de substances (chimiques) ou micro-organismes nocifs; les travaux soumis à un système d'organisation du temps de travail contraignant (art. 62 OLT 1).

\section{Versement du salaire pendant la grossesse et} après la naissance (ch. $8.1 \mathrm{ss}$ du contrat type)

Il faut distinguer entre deux phases: la première, la grossesse, au cours de laquelle la poursuite du versement du salaire s'appuie sur l'art. 324a du Code des obligations (CO), et la seconde, la période après la naissance, qui suit les dispositions de la loi sur les allocations pour perte de gain (LAPG). Consultez à ce sujet le complément à la solution légale proposé à l'art. 8.3 al. 2 du contrattype.

Le droit à une allocation de maternité commence le jour de la naissance et se termine au plus tard après 14 semaines, soit 98 jours. En cas d'hospitalisation prolongée de l'enfant, la mère peut demander que le droit à l'allocation commence à la date du retour de l'enfant à la maison. Versée par la caisse de compensation AVS, l'allocation de maternité est une indemnité journalière qui s'élève à $80 \%$ du revenu moyen perçu avant la naissance et au plus à 196 francs par jour.

Lorsque l'employeur a contracté une assurance d'indemnité journalière, l'employée peut faire valoir auprès de celle-ci son droit de recevoir la différence entre les 196 francs mentionnés et l'indemnisation à la charge de l'assurance ( $80 \%$ du salaire assuré), pour la période de la maladie, et au plus jusqu'au terme du congé maternité. Si l'employée est encore malade après la fin du congé maternité, elle a droit à toute l'indemnité journalière de l'assurance indemnité journalière.

Tant qu'une allocation de maternité est versée à une employée, celle-ci ne peut pas toucher les indemnités journalières de l'assurance-chômage, de l'AI ou de l'assurance-accidents obligatoire. Les indemnités de l'assurance indemnité journalière et de l'assurance-accidents obligatoire ne sont pas soumises aux cotisations AVS, en revanche les prestations perçues conformément à la LAPG le sont.

\section{Contrats de travail et autres conditions d'engagement}

Nous conseillons vivement d'établir le contrat par écrit, sur la formule de la FMH créée conjointement avec les associations des assistantes médicales, accompagnée des recommandations cantonales. Ces documents peuvent être obtenus aux adresses suivantes:

- Fédération des médecins suisses (FMH), Elfenstrasse 18, case postale 300,3000 Berne 15, tél. 031 35911 11, www.fmh.ch, e-mail: mpa[at]fmh.ch

- Association romande des assistantes médicales ARAM, 1003 Lausanne, tél. 0793801244 / 079471 68 53, www.aram-vd.ch; e-mail: info[at]aram-vd.ch 
- Schweizerischer Verband Medizinischer PraxisFachpersonen SVA, Monbijoustrasse 35, case postale, 3001 Berne, tél. 03138054 54, www.sva.ch, e-mail: sekretariat[at]sva.ch

Les Associations des assistantes médicales du canton de Genève et du Tessin ont leur propre convention de travail ou contrat de travail; leurs adresses sont les suivantes:

- Associazione Ticinese Assistenti di studio Medico ATAM, c/o M. Carrera, Via dei Faggi 2 A, 6912 LuganoPazzallo, www.atam.ch, e-mail: atam[at]bluewin.ch

- Association genevoise des assistantes médicales AGAM, 1200 Genève, www.agam-ge.ch, e-mail: presidente[at]agam-ge.ch
Les salaires des personnes en formation se basent sur les recommandations cantonales.

- Délégués aux questions des assistantes médicales: Dr Adrian Sury, président / Dr Renato Tognina, viceprésident

- Association genevoise des assistantes médicales AGAM: Régine Frossard, présidente

- Assoziazione Ticinese Assistenti di studio Medico ATAM: Mary Carrera, Segretaria

- Association romande des assistantes médicales ARAM: Marie-Paule Fauchère, présidente

- Schweizerischer Verband Medizinischer PraxisFachpersonen SVA: $\mathrm{M}^{\mathrm{e}}$ Bruno Gutknecht, secrétaire central 